\title{
AÇÕES INTERVENCIONISTAS NO ALIVIO DOS SINTOMAS DE DOENÇAS REUMÁTICAS EM IDOSOS
}

\section{INTERVENTIONAL ACTIONS IN THE RELIEF OF SYMPTOMS OF RHEUMATIC DISEASES IN THE ELDERLY}

\author{
Paula Moura Oliveira ${ }^{1}$ \\ Luan Felix Silva Alves ${ }^{2}$ \\ Ariane Gomes da Silva ${ }^{3}$ \\ Natam Oliveira Pires ${ }^{4}$ \\ Thaís Ribeiro Nascimento ${ }^{5}$ \\ Graciete Souza Cruz ${ }^{6}$ \\ Luzia Wilma Santana da Silva ${ }^{7}$ \\ Tatiane Dias Casimiro Valença ${ }^{8}$
}

Resumo: O Brasil tem apresentado nas últimas décadas o aumento da população idosa e da incidência das doenças crônicas não transmissíveis, como as reumáticas, que comprometem a capacidade funcional das pessoas idosas. O objetivo do estudo foi traçar o perfil sociobiodemográfico e a partir desse conhecimento elaborar um programa de exercícios físicos para alívio dos sintomas apresentados pelos idosos com doenças reumáticas cadastrados em um projeto de extensão. Estudo quantitativo, exploratório, descritivo e intervencionista realizado com 40 idosos cadastrados em um Núcleo de Extensão da Universidade Estadual do Sudoeste da Bahia (UESB), Jequié-BA. Foi empregado um questionário sociobiodemográfico elaborado pelos pesquisadores e uma abordagem intervencionista com programa de exercício físico funcional. Os dados foram analisados por meio do Software Statistical Package for Social Science (SPSS) ${ }^{\circledR}$ versão 20.0. Os resultados foram de $100 \%$ mulheres, $70 \%$ entre $60-70$ anos, $75 \%$ cor parda, $65 \%$ donas de casa, $25 \%$ ensino fundamental I incompleto, 95\% renda de 1 a 3 salários mínimos, $100 \%$ com osteoartrite, $75 \%$ apresentavam HAS, $20 \%$ deram nota 8 e 20\%, nota 5 para a dor, $70 \%$, acometidos na região da coluna lombar. A literatura científica indica a prática de exercício físico como uma das condutas mais eficaz no tratamento das doenças reumáticas. Concluiu-se que o conhecimento do perfil dos idosos possibilitou caracterizar as doenças reumáticas e os sintomas que afetavam os idosos cadastrados no projeto de extensão, assim como a elaboração de um programa de

1 Graduada em Fisioterapia pela Universidade Estadual do Sudoeste da Bahia (UESB), E-mail: paula.fisio.01@gmail.com.

${ }^{2}$ Graduado em Fisioterapia pela Universidade Estadual do Sudoeste da Bahia (UESB), E-mail: luan.fisiorp@gmail.com.

${ }^{3}$ Graduada em Fisioterapia pela Universidade Estadual do Sudoeste da Bahia (UESB), E-mail: ari-gomes@live.com.

${ }^{4}$ Graduado em Fisioterapia pela Universidade Estadual do Sudoeste da Bahia (UESB), E-mail: natan.pires93@gmail.com.

${ }^{5}$ Graduada em Fisioterapia pela Universidade Estadual do Sudoeste da Bahia (UESB), E-mail: thaisrn.fisioterapia@gmail.com.

${ }^{6}$ Graduada em Fisioterapia pela Universidade Estadual do Sudoeste da Bahia (UESB), E-mail: gscruz.fisio@hotmail.com.

7 Pós-doutorado em Enfermagem (UFSC). Enfermeira. Docente Pleno do Curso de Graduação em Enfermagem da Universidade Estadual do Sudoeste da Bahia (UESB), membro do Departamento de Saúde 2.

8 Doutorado em Memória pela Universidade Estadual do Sudoeste da Bahia (UESB), E-mail: tativalenca26@gmail.com. 
exercício físico que visou a melhora dos sintomas das doenças reumáticas nesse grupo de idosos.

Palavras-chave: Pessoa idosa. Doenças reumáticas. Sinais e sintomas. Exercício físico.

\begin{abstract}
In recent decades, Brazil has shown an increase in the elderly population and the incidence of chronic non-communicable diseases, such as rheumatic diseases, which compromise the functional capacity of the elderly. The aim of the study was to outline the sociobiodemographic profile and, based on this knowledge, develop a physical exercise program to relieve the symptoms presented by the elderly with rheumatic diseases registered in an extension project. Quantitative, exploratory, descriptive and interventional study performed with 40 elderly people registered at an Extension Center at the State University of Southwest Bahia (UESB), Jequié-BA. A sociobiodemographic questionnaire developed by the researchers and an interventionist approach with a functional physical exercise program were used. The data were analyzed using the Software Statistical Package for Social Science (SPSS)® version 20.0 . The results were $100 \%$ women, $70 \%$ between $60-70$ years old, $75 \%$ brown, $65 \%$ housewives, $25 \%$ incomplete elementary school I, $95 \%$ income from 1 to 3 minimum wages, 100\% with osteoarthritis, $75 \%$ had $\mathrm{SAH}, 20 \%$ scored 8 and $20 \%$ scored 5 for pain, $70 \%$ affected in the lumbar spine. The scientific literature indicates the practice of physical exercise as one of the most effective approaches in the treatment of rheumatic diseases. It was concluded that the knowledge of the profile of the elderly made it possible to characterize rheumatic diseases and the symptoms that affected the elderly registered in the extension project, as well as the elaboration of a physical exercise program aimed at improving the symptoms of rheumatic diseases in this group of senior people.
\end{abstract}

Key words: Elderly person. Rheumatic diseases. Signals and symptoms. Physical exercise.

Data de submissão: 02.07.2019

Data de aprovação: 18.12.2020

Identificação e disponibilidade:

(https://revista.univap.br/index.php/revistaunivap/article/view/2322,

http://dx.doi.org/10.18066/revistaunivap.v27i53.2322).

\title{
1 INTRODUÇÃO
}

Análise sobre dados demográficos evidencia acréscimo em números de pessoas idosas em nível mundial. Neste cenário, o Brasil a cada década apresentase com significativo aumento como divulgado pelo Instituto Brasileiro de Geografia e Estatística (INSTITUTO..., 2018), ao enunciar que em 2060 o número de pessoas com 65 anos ou mais poderá atingir a marca de $25,5 \%$ da população, o que representa cerca de 58,2 milhões de idosos na pirâmide demográfica do país.

Este cenário pode ser explicado por alguns fatores, como a redução das taxas de fecundidade e de mortalidade associadas ao aumento da expectativa de vida das pessoas, além do desenvolvimento na área do conhecimento, práticas e tecnologias em saúde (MIRANDA; MENDES; SILVA, 2016; QUEIROZ et al., 2015; KUNZLER; 
BULLA, 2014). Apesar disto, ainda boa parte das pessoas idosas estão envelhecendo com baixa qualidade de vida e em condição de doenças crônicas não transmissíveis (DCNT) e suas consequências (KENNEDY et al., 2014).

Para Santos e colaboradores (2015), a conquista do envelhecer alcançada ao longo dos anos se transformou em um grande desafio para o século atual. Isto devido às demandas de cuidado à população idosa que apresenta alterações fisiológicas e funcionais próprias do processo natural de envelhecimento e as sujeitam ao desenvolvimento de Doenças Crônicas não Transmissíveis (DCNT) que são mais frequentes nessa faixa etária. Não que o envelhecer signifique necessariamente adoecer, mas a associação desse processo com a presença de doenças crônicas pode comprometer o envelhecimento ativo e saudável (MIRANDA; MENDES; SILVA, 2016).

As DCNT podem provocar alterações degenerativas em diferentes tecidos do corpo humano gerando problemas graves à saúde, como alterações na capacidade funcional, dependência e redução dos dias de vida, e são responsáveis por aproximadamente $70 \%$ de todas as mortes no mundo (BRASIL, 2011). Trata-se de doenças que são influenciadas por fatores como tabagismo, alcoolismo, consumo abusivo do álcool, hábitos alimentares inadequados e sedentarismo (MALTA et al., 2017). A intervenção nesses fatores mostra-se como fundamental, pois, poderia resultar em redução do número de comorbidades e de mortes prematuras, por Acidente Vascular Encefálico (AVE), Diabetes Mellitus (DM), Hipertensão Arterial Sistêmica (HAS), doenças respiratórias, cardiovasculares, neurológicas, reumáticas e câncer que se encontram entre as mais prevalentes (MALTA et al., 2017, BRASIL, 2014).

Entre as DCNT estão as doenças reumáticas, descritas como um conjunto de enfermidades, sistêmicas e de características autoimunes, com especial comprometimento das articulações sinoviais, e não apenas, as articulares e periarticulares, mas também outros órgãos, como a pele, coração, pulmão, fígado e rins (REBELLO et al., 2015; MARTÍN et al., 2014). Estando a osteoartrite, artrite reumatoide, osteoporose, fibromialgia, lúpus eritematoso sistêmico, espondilite anquilosante, gota, febre reumática, reumatismo de partes moles como as mais frequentes, entre outras (REBELLO et al., 2015; MARTíN et al., 2014).

Os sintomas e as consequências osteomioarticulares ocasionados pela presença das doenças reumáticas podem comprometer a realização de atividades diárias e instrumentais dos indivíduos (NAGAYOSHI et al., 2018; FRANCISCO et al., 2018). Para os quais é necessário um trabalho em equipe multiprofissional ao tratamento que dependerá do grau de comprometimento da doença. Entre as formas de tratamento estão o acompanhamento médico com a prescrição de medicamentos para alivio dos sintomas e controle da doença; fisioterápico para melhora dos sintomas, prevenção de deformidades, ganho e manutenção da força, resistência e equilíbrio, e, aumento da funcionalidade, e outros; apoio psicológico; atividade física regular entre outros (REBELLO et al., 2015; DUARTE et al., 2013).

$\mathrm{Na}$ literatura, estudos sobre o tratamento das doenças reumáticas têm demonstrado ser a prática do exercício físico eficaz, por sua ação diretamente na minimização da percepção dolorosa, diminuição do espasmo muscular, manutenção e ganho da amplitude de movimento articular, aumento da força muscular, condicionamento cardiorrespiratório, melhora da circulação, ganho de equilíbrio e coordenação (JORGE et al., 2018; BARDUZZI et al., 2013). Além disso, por promover interação social, melhora do humor e da sensação de bem estar, principalmente quando realizada em grupo (BARDUZZI et al., 2013). 
Neste particular, exercícios físicos regulares para pessoas idosas com doenças reumáticas torna-se uma estratégia à funcionalidade e qualidade de vida deste grupo etário. Igualmente, no que tange aos profissionais da área de fisioterapia imergir sobre estes saberes potencializa o direcionamento dos quefazeres de cuidados à inclusão de exercícios físicos em sua prática clínica avançando para além do tratamento conservador.

A partir das reflexões realizadas surgiram as perguntas: é possível caracterizar as doenças osteoarticulares e os sintomas predominantes nos idosos cadastrados em um projeto de extensão? É possível elaborar um programa de exercícios físicos específico para esse grupo de idosos que apresentam essas doenças?

Sendo assim, o estudo que se apresenta teve como objetivo traçar o perfil sociobiodemográfico e a partir desse conhecimento elaborar um programa de exercícios físicos para alívio dos sintomas apresentados pelos idosos com doenças reumáticas cadastrados em um projeto de extensão.

\section{METODOLOGIA}

Estudo quantitativo, exploratório, descritivo e intervencionista, realizado no período de abril a dezembro/2018, com 40 idosos (a partir de 60 anos de idade) cadastrados no Núcleo Interdisciplinar de Estudos e Extensão em Cuidados à Saúde da Família em Convibilidade com Doenças Crônica (NIEFAM), da Universidade Estadual do Sudoeste da Bahia (UESB), campus de Jequié-BA, segundo os critérios de inclusão: idoso em condição cognitiva para responder ao questionário e cadastrado no NIEFAM; apresentar diagnóstico médico comprobatório de doenças reumáticas; sintomas de dor devido alguma doença reumática e, ser anuente à pesquisa. Os critérios de exclusão foram: idosos com sintomas, mas sem comprovação de diagnóstico médico; ausência em mais de $25 \%$ das intervenções.

A coleta dos dados foi desenvolvida pelos pesquisadores mediante um questionário de avaliação sociobiodemográfica composto de duas seções: 1a. de informações sociodemográficas, como nome, sexo, idade, raça, estado civil, ocupação, com quem reside, renda familiar, atividades de lazer; e a $2^{\mathrm{a}}$. com informações clínicas relacionadas ao histórico de doenças reumáticas: doença diagnosticada, tempo de diagnóstico, queixa principal, o que melhora e o que piora os sintomas, deformidades osteoarticulares, avaliação da dor, local da dor, outras patologias associadas, evento de queda e postura na atividade laboral. Este instrumento foi aplicado a cada idoso individualmente, em uma sala do Centro Social Urbano de Jequié-BA (CSU), local no qual são desenvolvidas as atividades do NIEFAM e teve duração média de 30 minutos.

O processo de conhecer as condições de vida e saúde dos idosos teve o objetivo de delinear um plano de intervenção às demandas identificadas, o qual se desenvolveu com a aplicação de exercícios físicos funcionais, realizados três vezes por semana (segundas, quartas e sextas feiras), com duração de 60 minutos, no período de abril a dezembro/2018. As intervenções se concentraram na realização de exercícios de alongamento (durante 10 minutos), aquecimento muscular (duração de 5 minutos), fortalecimento muscular de Membros inferiores e superiores com número de séries e repetições (3 séries de 10 repetições cada tipo de exercício), coordenação motora e equilíbrio(como andar em linha reta, para frente e para trás, em "zig zag", ficar em pé em apoio uni podal), respiratórios (respiração profunda e fracionada em tempos) e funcionais (subida e descida em "step", agachamento, sentar e levantar da cadeira, afundo, entre outros) durante o período de 35 minutos. 
Em seguida foram realizados alongamentos globais e relaxamento (por 10 minutos). Para isso foram utilizados como materiais: halteres, caneleiras, bola suíça, cones, theraband, tatames, bastões e cadeiras. Ao final do período de intervenção, outro instrumento foi inserido a abordagem investigativa e tangenciou sobre uma pergunta aberta em profundidade a respeito de o quão a intervenção teria influenciado nos sintomas osteomioarticulares dos participantes.

Os dados coletados foram processados no Software Statistical Package for Social Science ${ }^{\circledR}$ (SPSS) versão 20.0 e submetidos à análise descritiva das variáveis categóricas apresentando os resultados pelas frequências absolutas e relativas.

Este estudo foi aprovado por Comitê de Ética em Pesquisa em observância a Resolução 466/12 (BRASIL, 2012), conforme Parecer no 639.056, CAAE 27221414.3.0000.0055.

\section{RESULTADOS}

Os resultados evidenciam uma população de predominância feminina (100\%), que apresentou faixa etária dos 60-70 anos (70\%) e de cor parda (75\%). Mais da metade casada (55\%), tendo como ocupação os cuidados da casa $(65 \%)$. Em relação à escolaridade predominou a formação em ensino fundamental I incompleto $(25 \%)$ e referente à renda familiar esta foi de 1 a 3 salários mínimos (95\%), conforme exibido na Tabela 1.

Tabela 1 - Distribuição dos idosos segundo as características sociodemográficas. Jequié - BA, Brasil, 2018.

(continuação)

\begin{tabular}{lcc}
\hline \multicolumn{1}{c}{ Variáveis } & $\mathbf{n}$ & $\%$ \\
\hline Sexo & 40 & 100 \\
Feminino & 0 & 0 \\
Masculino & 28 & 70 \\
Idade & 12 & 30 \\
60-70 anos & & \\
Mais de 71 anos & 30 & 75 \\
Cor & 6 & 15 \\
Parda & 4 & 10 \\
Branca & & \\
Negra & 22 & 55 \\
Estado civil & 6 & 15 \\
Casado (a) & 6 & 15 \\
Viúvo (a) & 6 & 15 \\
Separado (a) & & \\
Solteiro (a) & 26 & 65 \\
Ocupação & 6 & 15 \\
Dona de casa & 2 & 5 \\
Costureira & 2 & 5 \\
Doméstica & 2 & 5 \\
Comerciante & 2 & 5 \\
Autônoma & & \\
Aposentada &
\end{tabular}


Tabela 1 - Distribuição dos idosos segundo as características

(conclusão) sociodemográficas. Jequié - BA, Brasil, 2018.

\begin{tabular}{lrc}
\hline Escolaridade & & 25 \\
Ensino fundamental I incompleto & 10 & 20 \\
Ensino fundamental II completo & 8 & 20 \\
Ensino médio completo & 8 & 20 \\
Ensino fundamental I completo & 8 & 5 \\
Ensino fundamental I Incompleto & 2 & 5 \\
Ensino médio incompleto & 2 & 5 \\
Analfabeto & 2 & \\
Renda familiar & 38 & 95 \\
De 1 a 3 salários mínimos & 2 & 5 \\
Mais de 3 salários mínimos & & \\
\hline
\end{tabular}

Fonte: Elaborado pelos autores. Dados da pesquisa (2018).

Em relação às características clínicas todas as idosas (100\%) apresentavam diagnóstico de osteoartrite (ou osteoartrose ou artrose), sendo que, a maioria (75\%) já apresentava esse diagnóstico há mais de um ano. A dor foi a queixa mais frequente, apontada por todas as participantes (100\%). Para maior parte delas a posição que melhorava o quadro álgico era a de repouso (60\%) e a que piorava era a de movimento para metade delas $(50 \%)$, sendo que uma parcela significativa trabalhava na posição em pé $(90 \%)$ e apresentava como outras doenças associadas à HAS (75\%) e ocorrências cirurgias (75\%), como exposto na Tabela 2.

Tabela 2 - Distribuição dos idosos segundo as características clínicas. (continuação) Jequié - BA, Brasil, 2018.

\begin{tabular}{lrc}
\hline \multicolumn{1}{c}{ Variáveis } & $\mathbf{n}$ & $\%$ \\
\hline Diagnóstico médico & & \\
Osteoartrose/ osteoartrite/Artrose & 40 & 100 \\
Artrite reumatoide & 8 & 20 \\
Osteoporose & 8 & 20 \\
Fibromialgia & 6 & 15 \\
Reumatismo partes moles & 4 & 10 \\
Espondilite anquilosante & 2 & 5 \\
Outros & 2 & 5 \\
Tempo de diagnóstico & 30 & 75 \\
Mais de um ano & 10 & 25 \\
Até um ano & & \\
Queixa principal & 40 & 100 \\
Dor & 18 & 45 \\
Formigamento & 12 & 30 \\
Dificuldade para andar & 12 & 30 \\
Fadiga & 6 & 15 \\
Tontura & 2 & 5 \\
Cefaleia & 12 & 60 \\
O que melhora a queixa & 6 & 30 \\
Repouso & & 50 \\
Movimento & 10 & \\
O que piora a queixa & & \\
Movimento & &
\end{tabular}


Tabela 2 - Distribuição dos idosos segundo as características clínicas. Jequié - BA, Brasil, 2018.

\begin{tabular}{lrc}
\hline Repouso & 7 & 35 \\
Posição de trabalho & 36 & 90 \\
Em Pé & 28 & 70 \\
Pegar peso & 24 & 60 \\
Inclinada & 10 & 25 \\
Sentada & 6 & 15 \\
Agachar & 4 & 10 \\
Movimentos repetitivos & & \\
Outras doenças & 30 & 75 \\
HAS & 30 & 75 \\
Cirurgias & 26 & 65 \\
Alterações visuais & 12 & 30 \\
Diabetes Mellitus & 6 & 15 \\
Doenças gastrointestinais & 6 & 15 \\
Doenças cardíacas & 2 & 5 \\
Doenças respiratórias & 2 & 5 \\
Outras & & \\
\hline
\end{tabular}

Fonte: Elaborado pelos Autores. Dados da pesquisa, 2018.

Os resultados apresentados na Tabela 3 revelam o grau de intensidade da dor avaliado a partir da Escala Visual Analógica da Dor (EVA) (MARTINEZ; GRASSI; MARQUES, 2011), a intensidade da dor variou de 5 a 10, sendo $8(20 \%)$ e $5(20 \%)$ as mais referidas pelas participantes. Em relação à localização da dor foi possível observar uma variedade de locais afetados por esse sintoma predominando a região da coluna lombar (70\%).

Tabela 3 - Distribuição dos idosos segundo a intensidade (EVA) e localização da dor. Jequié - BA, Brasil, 2018.

\begin{tabular}{lll}
\hline \multicolumn{1}{c}{ Variáveis } & $\mathrm{n}$ & $\%$ \\
\hline Intensidade da dor & 8 & 20 \\
Intensidade 8 & 8 & 20 \\
Intensidade 5 & 6 & 15 \\
Intensidade 10 & 6 & 15 \\
Intensidade 9 & 6 & 15 \\
Intensidade 7 & 6 & 15 \\
Intensidade 6 & & \\
Localização da dor & 28 & 70 \\
Coluna lombar & 10 & 25 \\
Joelho direito & 10 & 25 \\
Joelho esquerdo & 8 & 20 \\
Ombro direito & 6 & 15 \\
Ombro esquerdo & 4 & 10 \\
Coluna cervical & 4 & 10 \\
Punho Direito & 2 & 5 \\
Punho esquerdo & 2 & 5 \\
Mão direita & 2 & 5 \\
Cotovelo direito & 1 & 5 \\
Coluna torácica & & 5 \\
\hline
\end{tabular}

Fonte: Elaborado pelos Autores. Dados da pesquisa (2018). 


\section{DISCUSSÃO}

Os resultados revelaram uma predominância de participantes do sexo feminino. Esse mesmo resultado foi observado em outros estudos corroborando com a ideia da chamada feminização da velhice, uma vez que é notória a presença das mulheres, principalmente, na faixa etária em foco, tanto em nível mundial como no Brasil (MANSO; GALERA, 2015; MANSO; RIBEIRO, 2012). Dados demográficos revelaram que as mulheres são maioria expressiva na população idosa brasileira com 16,9 milhões (56\%), enquanto homens idosos representam 13,3 milhões (44\%) (INSTITUTO..., 2018).

A incidência da osteoartrite em mulheres tem relação com o estrogênio, um importante hormônio que desempenha papel na manutenção da homeostase dos tecidos articulares (PACCA et al., 2018), que na fase da menopausa, geralmente entre 45-55 anos de idade, há redução em sua produção de forma progressiva, acarretando perdas funcionais, a exemplo do tecido ósseo, com aumento da atividade dos osteoblastos, inibição da retirada de cálcio do organismo e diminuição da formação e atividade dos osteoclastos, degeneração articular, entre outras (WOLFF et al., 2012).

Em relação à idade, encontravam-se na faixa etária dos 60-70 anos, exatamente ao período do ciclo vital em que o organismo está mais exposto a desgastes de natureza fisiológica, razão pela qual estudos são categóricos ao enunciarem que o envelhecimento é um dos principais fatores de risco para o desenvolvimento da maioria das DCNT (PACCA, et al., 2018; REZENDE; CAMPOS; PAILO, 2013), uma vez que o organismo se torna mais vulnerável. Assim, autores como Pacca, et al., (2018) e Rezende, Campos e Pailo (2013), afirmam que há uma forte tendência em aumentar a incidência da osteoartrose no país, uma vez que a população está ficando cada vez mais velha. A respeito disso os dados demográficos evidenciaram que $40 \%$ da população brasileira com idade acima de 70 anos são acometidas por osteoartrose e cerca de $80 \%$ com algum tipo de limitação de movimento ou dificuldades para a realização de suas atividades de vida diária (AVDs) (LEITE et al., 2011).

Concernente ao estado civil, foi observado que a maioria das idosas é casada, dado que também encontra aderência noutras pesquisas no cenário nacional e que transversaliza aspectos de funcionalidade na presença de DCNT em pessoas idosas (REIS et al., 2019). Este fato acena uma especial relevância, tendo em vista que os casos de DCNT podem evoluir para um quadro de dependência, razão pela qual se torna importante à relação conjugal por ter a possibilidade de corresponsabilidade nos cuidados de saúde no sistema familiar e de saúde.

Tratando da escolaridade os resultados apontam baixo nível de formação, o que reflete o cenário nacional em número de pessoas idosas iletradas e que pontua um problema de saúde pública, pois o analfabetismo tem se tornado uma fonte de preocupação para os profissionais de saúde, uma vez que a ausência ou a baixa escolaridade promovem maiores dificuldades de acesso das pessoas às redes de cuidados e assimilação de conhecimentos a respeito de sua condição de saúde (SILVA et al., 2017). Situação que, segundo estudiosos, pode ocasionar um maior declínio na capacidade funcional e na qualidade de vida, principalmente daquelas pessoas que necessitam de maior acompanhamento da equipe de saúde (LOPES; SANTOS, 2015).

Quanto à ocupação a maioria se dedicava ao cuidado do lar, com renda familiar variando entre um e três salários mínimos e principal fonte de entrada a 
aposentadoria. Este dado pode ainda ser mais bem compreendido ao correlacioná-lo às DCNT, por estas serem geradoras potenciais de limitações, o que dificulta ou impede de certa maneira de as pessoas acometidas executarem atividades laborais, impactando em afastamento do mercado de trabalho ou em aposentadorias por invalidez, o que acarreta perdas econômicas e sociais, sendo destacados os custos com medicamentos, planos de saúde e sustento próprio e da família são elevados em relação ao que recebem (MARCHI et al., 2013).

A totalidade das participantes do estudo apresentou osteoartrite, sendo que a maioria foi diagnosticada há mais de ano, dado ratificado na literatura, sobre a doença acometer preferencialmente o sexo feminino (JORGE et al., 2018). Outro fator relacionado, trata-se de a patologia atingir as pessoas em idade mais avançada (JORGE et al., 2018; REZENDE; CAMPOS; PAILO, 2013; LEITE et al., 2011), o que vem corroborar os dados estatísticos de a osteoartrite ser a doença reumática mais comum na população brasileira, acometendo entre $44 \%$ a $70 \%$ das pessoas acima dos 50 anos de idade podendo chegar a $85 \%$ na faixa etária acima de 75 anos (LOURESA et al., 2016; DUARTE et al., 2013; LEITE et al., 2011).

Quanto ao diagnóstico da osteoartrite, muitas vezes, acontece com o quadro já bem evoluído de comprometimento da cartilagem, uma vez que os sintomas só vão se manifestar em fases mais avançadas da doença, que evolui de maneira crônica e progressiva com degeneração da cartilagem articular e até comprometimento do osso subcondral e de outros tecidos periarticulares (SOUZA et al., 2017). Dado que foi observado nesse estudo, em que um número significativo de idosas já conviviam com a doença por um tempo prolongado.

Os sintomas podem variar em função da gravidade das lesões sendo que à medida que a doença evolui, a dor pode aparecer ou se exacerbar a pequenos esforços ou até mesmo estar presente com a pessoa em repouso (DUARTE et al., 2013), como foi observado neste estudo, sobre a dor melhorar com o repouso e piorar com o movimento, segundo os relatos das participantes. Ressalta-se que o movimento aqui seria o realizado de forma repetitiva ou com sobrecarga ou realizado da maneira incorreta e não a atividade física que visa o movimento adequado e outros benefícios em nível articulares e musculares. Assim, a evolução da osteoartrose precisa ser avaliada e classificada de acordo com o grau de comprometimento articular radiográfico para auxiliar os profissionais da saúde na tomada de decisão em relação ao tratamento mais adequado ao quadro que o paciente apresenta (MARTINS et al., 2017), assim como a prescrição dos exercícios físicos regulares.

Quanto à localização da dor, a coluna lombar e os joelhos foram as regiões mais afetadas pelos sintomas de acordo com as participantes. Resultados semelhantes foram encontrados nos estudos de Vieira et al., (2012) e Jorge et al., (2018). Para Duarte et al., (2013), fatores como a idade, a genética e a presença de outras doenças articulares contribuem para afetar as estruturas articulares influenciando a localização e a severidade do desenvolvimento da osteoartrose em uma articulação. Nesse estudo a maior parte das participantes referiu trabalhar em posição de pé, sendo essa uma postura que gera grande sobrecarga, principalmente nas articulações do joelho e coluna lombar, e, a depender da velocidade, frequência e tipo de movimento realizado pode gerar risco ainda maior de lesão na cartilagem e em outras estruturas periarticulares.

A queixa principal da totalidade das participantes foi a dor. Quando questionadas a respeito da intensidade desta, os valores numéricos atribuídos mais frequentes foram 8 e 5 (20\%, Tabela 3) na EVA, principalmente às regiões da coluna 
lombar e dos joelhos. A literatura revela que entre os principais sinais e sintomas da osteoartrose estão as dores musculoesqueléticas, sinovite, edema, rigidez articular matinal, crepitação óssea, diminuição da amplitude de movimento articular e atrofia muscular. Estes sintomas podem acarretar dificuldades na realização das atividades do cotidiano, gerando dependência, diminuição do bem-estar e da qualidade de vida das pessoas acometidas (JORGE et al., 2018; DUARTE et al., 2013). Estudos científicos demonstraram que uma vida ativa com a adoção de atividade física regular se reverte em uma das melhores formas de tratar os sintomas das doenças reumáticas (SANTANA, et al., 2014; FERNANDES; NOGUEIRA, 2016; DUARTE et al., 2013).

A respeito da adoção de exercício físico e melhora da dor Santana et al., (2014) constatou que o exercício resistido, realizado duas a três vezes por semana, com série de oito a doze repetições, com duração de pelo menos trinta minutos, são muito eficazes na redução da dor. Além disso, a prática do exercício físico melhora a resistência cardiorrespiratória e muscular do indivíduo promovendo alterações nos circuitos neurais que modulam a dor (ELLINGSON et al., 2012).

O estudo de Fernandes e Nogueira (2016) demonstrou que a criação e implantação de um protocolo de atividade física domiciliar para pacientes com osteoartrite graus III, IV e V, segundo a classificação de Ahlback, se mostrou eficiente para a melhora da dor dos 23 participantes da pesquisa. Também Oliveira, Vatri e Alfieri (2016) constataram a partir de treinamento com exercícios de alongamento e fortalecimento muscular resistido para 30 pacientes com osteoartrite em joelhos que houve melhora da dor, rigidez articular, funcionalidade, mobilidade funcional e força. Outra abordagem promotora da melhora na percepção dolorosa é a hidroterapia que apresenta resultados significantes, como observado no estudo de Barduzzi et al., (2013) em idosos com osteoartrite de joelho submetidos a um protocolo de exercício na água, cujo os resultados demonstraram redução do tempo da marcha usual, marcha rápida e de subir e descer escadas.

A presença da dor crônica, a dificuldade para realização das atividades da vida diária, restrição na mobilidade, dependência para realização das AVDs, afastamento do trabalho e outros problemas gerados pelas osteoartrites podem favorecer 0 aparecimento da ansiedade, promover o desânimo e desencadear quadro de depressão e isolamento social. A respeito disto, Vieira et al., (2012) constataram que a adoção de atividade física, principalmente em grupo contribui positivamente para a prevenção e o alivio dos sintomas físicos, sociais e emocionais dos pacientes, os quais obtiveram melhoras nos quesitos de qualidade de vida, capacidade funcional, limitação física, dor, vitalidade, aspectos sociais, emocionais e saúde mental.

Assim, atividades que vão ao encontro desta abordagem são necessárias e de potencialização do longe viver, no que o estudo em foco perseguiu as participantes. Igualmente, ao perspectivar outras comorbidades que as afetavam - HAS e os casos de cirurgia -, estas as mais apontadas pelas participantes. Sobre isto, Leite et al., (2011), também atentaram sobre a frequência de tais doenças em seu estudo, no qual a HAS foi de $75,8 \%$ da amostra. O que remete ao entendimento do olhar sistêmico sobre o envelhecimento humano, exemplificado pelo fato de tanto a osteoartrite como a HAS apresentam-se como fator de risco a idade aumentada (MATAVELLI et al., 2014; LEITE et al., 2011). Além disso, a HAS é uma doença bastante frequente na população brasileira devido a resistência dos pacientes em aderirem ao tratamento, hábitos de vida impróprios, como o sedentarismo, alimentação rica em gorduras saturadas, tabagismo, alcoolismo, estresse, entre outros (SOCIEDADE BRASILEIRA DE CARDIOLOGIA, 2016). 
De acordo com Leite et al. (2011), a presença de comorbidades como a HAS, DM, AVE associadas a osteoartrite pode convergir para o agravamento da incapacidade física e a dependência funcional, cujo impacto estará diretamente na condição de saúde e na qualidade de vida dos pacientes.

A análise dos resultados a respeito da presença da osteoartrite e HAS na população estudada evidencia que a adoção de um programa de atividade física é fundamental para o controle e tratamento das ambas as doenças, a exemplo do que enuncia a $7^{\text {a }}$ Diretriz Brasileira de Hipertensão Arterial (SOCIEDADE BRASILEIRA DE CARDIOLOGIA, 2016), de que, além do tratamento medicamentoso para a HAS, deve ser adotado o tratamento não medicamentoso que previne os fatores de risco. Outros estudiosos se somam a esta compreensão ao destacarem que além da prática de atividade física regular, devem-se incluir medidas nutricionais, controle do peso, parar de fumar, controlar o estresse entre outros (MATAVELLI et al., 2014; LEITE et al., 2011).

Não obstante, ressalta-se haver limitações neste estudo, que se assentam na falta de equipamentos para mensuração de parâmetros mais específicos à avaliação da força muscular, amplitude de movimento, volumes pulmonares, o que se mostra como necessário à realização de outras pesquisas para que tais marcadores sejam mais bem avaliados de maneira aos saberes sobre os benefícios de intervenção com atividade física nos sintomas e na capacidade funcional de pessoas idosas com DCNT.

\section{CONCLUSÃO}

A partir da aplicação do questionário foi possível caracterizar as doenças reumáticas e os sintomas que afetavam os idosos cadastrados no projeto de extensão. A partir das informações coletadas apoiadas nos estudos científicos que indicavam a atividade física como uma das principais formas de tratamento para essas doenças foi possível a elaboração de um programa de atividade física visando a melhora dos sintomas das doenças reumáticas nesse grupo de idosos.

\section{REFERÊNCIAS}

BARDUZZI, G. O. et al. Capacidade funcional de idosos com osteoartrite submetidos a fisioterapia aquática e terrestre. Fisioter. Mov., v. 26, n. 2, p. 349-60, 2013.

BRASIL. Conselho Nacional de Saúde. Resolução no 466, de 12 de dezembro de 2012. Aprova diretrizes e normas regulamentadoras de pesquisas envolvendo seres humanos. Diário Oficial da União, Brasília, DF, 2012. Disponível em: http://bvsms.saude.gov.br/bvs/saudelegis/cns/2013/res0466_12_12_2012.html Acesso em: 05 maio 2015.

BRASIL. Ministério da Saúde. Percepção do estado de saúde, estilos de vida e doenças crônicas: Brasil, Grandes Regiões e Unidades da Federação. 105 p. 2014. Disponível em:

http://www.dive.sc.gov.br/conteudos/agravos/publicacoes/PNS\%202013\%20Percepç ão\%20do\%20estado\%20de\%20saúde\%20estilos\%20de\%20vida\%20e\%20doenças 
\%20crônicas.pdf. Acesso: 25 maio 2019.

BRASIL. Ministério da Saúde. Plano de ações estratégicas para o enfrentamento das doenças crônicas não transmissíveis (DCNT) no Brasil 2011-2022. Brasília, DF: Ministério da Saúde, 2011. Disponível em:

http://bvsms.saude.gov.br/bvs/publicacoes/plano_acoes_enfrent_dcnt_2011.pdf Acesso: 29 maio 2019.

DUARTE, V. S. et al. Exercícios físicos e osteoartrose: uma revisão sistemática. Fisioter. Mov., v. 26, n. 1, p. 193-202, 2013.

ELLINGSON, L. D. et al. Physical activity, sustained sedentary behavior, and pain modulation in women with fibromyalgia. The Journal of Pain, v. 13, n. 2, p.195-206, 2012.

FERNANDES, R. S. C.; NOGUEIRA, M. P. Efeitos da orientação da atividade física em pacientes com osteoartrite avançada do joelho. Rev Bras Med Esporte, v. 22, n. 4 p. 302-305, 2016.

FRANCISCO, P. M. S. B. et al. Incapacidade funcional para atividades instrumentais da vida diária em idosos com doenças reumáticas. Rev. Bras. Geriatr. Gerontol., v. 21 , n. 5, p. 591-600, 2018.

INSTITUTO BRASILEIRO DE GEOGRAFIA E ESTATÍSTICA (IBGE). Número de idosos cresce 18\% em 5 anos e ultrapassa 30 milhões em 2017. 2018.

Disponível em: https://agenciadenoticias.ibge.gov.br/agencia-noticias/2012-agenciade-noticias/noticias/20980-numero-de-idosos-cresce-18-em-5-anos-e-ultrapassa-30milhoes-em-2017. Acesso: 25 maio 2019.

JORGE, M. S. G. et al. Efeitos da cinesioterapia na osteoartrite de joelho em idosos: revisão sistemática. ConScientia e Saúde, v. 7, n. 1, p. 93-100, 2018.

KENNEDY, B. K. K. et al. Geroscience: Linking Aging to Chronic Disease. Cell, v.159, p. 709-713, 2014.

KUNZLER, R. B.; BULLA, L. C. Idosos brasileiros: o contexto dos direitos sociais e das políticas sociais. Argumentum, v. 6, n.1, p. 153-59, 2014.

LEITE, A. A. et al. Comorbidades em pacientes com osteoartrite: frequência e impacto na dor e na função física. Rev Bras Reumatol, v. 51, n. 2, p. 113-123, 2011.

LOPES, G. L.; SANTOS, M. I. P. O. Funcionalidade de idosos cadastrados em uma unidade da Estratégia Saúde da Família segundo categorias da Classificação Internacional de Funcionalidade. Rev. Bras. Geriatr. Gerontol., v.18, n.1, p.71-83, 2015.

LOURESA, F. B. et al. Avaliação do índice de massa corporal como fator prognóstico na osteoartrose do joelho. Rev. Bras. ortop., v. 51, n. 4, p. 400-404, 2016.

MALTA, D. C. et al. Doenças crônicas não transmissíveis e a utilização de serviços 
de saúde: análise da Pesquisa Nacional de Saúde no Brasil. Rev. Saude Publica, v.51, Supl. 1, 4s, 2017.

MANSO, M. E. G.; GALERA, P. B. Perfil de um grupo de idosos participantes de um programa de prevenção de doenças crônicas. Estud. interdiscipl. envelhec., v. 20, n. 1, p. 57-71, 2015.

MANSO, M. E. G.; RIBEIRO, M. P. Caracterização das condições de saúde de um grupo de idosos pertencente a um plano de saúde. Revista Brasileira de Medicina, v. 69 , n. 3, p. $45-55,2012$.

MARCHI, K. C. et al. Adesão à medicação em pacientes com doença de Parkinson atendidos em ambulatório especializado. Ciência \& Saúde Coletiva, v. 18, n. 3, p. 855-862, 2013.

MARTíN, A. D. H. et al. Rehabilitación integral del paciente reumático. Revista Cubana de Reumatología, v. 16, n. 1, p. 15-22, 2014.

MARTINEZ, J. E.; GRASSI D. C.; MARQUES, L. G. Análise da aplicabilidade de três instrumentos de avaliação de dor em distintas unidades de atendimento:

ambulatório, enfermaria e urgência. Rev Bras Reumatol., v. 51, n. 4, p.299-308, 2011.

MARTINS, G. C. et al. Correlação entre a classificação radiográfica de Ahlbäck e o estado de conservação do ligamento cruzado anterior em gonartrose primária. Rev. Bras. Ortop., v. 2, n. 1, p. 69-74, 2017.

MATAVELLI, I. S. et al. Hipertensão Arterial Sistêmica e a Prática Regular de Exercícios Físicos como Forma de Controle: Revisão de Literatura. Rev Bras. Cienc. Saúde, v. 18, n. 4, p. 359-366, 2014.

MIRANDA, G. M. D.; MENDES, A. C. G.; DA SILVA, A. L. A. O envelhecimento populacional brasileiro: desafios e consequências sociais atuais e futuras. Rev. Bras. Geriatr. Gerontol., v. 19, n. 3, p. 507-519, 2016.

NAGAYOSHI, B. A. et al. Artrite reumatoide: perfil de pacientes e sobrecarga de cuidadores. Rev. Bras. Geriatr. Gerontol., v. 21, n. 1, p. 45-54, 2018.

OLIVEIRA, N. C.; VATRI, S.; ALFIERI, F. M. Comparação dos efeitos de exercícios resistidos versus cinesioterapia na osteoartrite de joelho. Acta Fisiátr., v. 23, n. 1, p. 7-11, 2016.

PACCA, D. M. et al. Prevalência de dor articular e osteoartrite na população obesa brasileira. ABCD Arq Bras Cir Dig., v. 31, n. 1, e1344, 2018.

QUEIROZ, M. A. C. et al. Social representations of sexuality for the elderly. Rev. Bras. Enferm., v. 68, n. 4, p. 662-667, 2015.

REBELLO, P. A. et al. Estamos Ensinando o que É Necessário sobre Doenças Reumatológicas? Revista Brasileira de Educação Médica, v. 39, n. 2, p. 246-251, 
2015.

REIS, J. W. S. et al. Condições de Saúde de Idosos com Diabetes Mellitus de uma Atenção Básica. Rev. Mult. Psic., v. 13, n. 45, p. 794-806, 2019.

REZENDE, M. U.; CAMPOS, G. C.; PAILO, A. F. Conceitos atuais em osteoartrite. Acta Ortop Bras., v. 21, n. 2, p. 120-122, 2013.

SANTANA, F. S. et al. Avaliação da capacidade funcional em pacientes com artrite reumatoide: implicações para a recomendação de exercícios físicos. Rev Bras. Reumatol., v. 54, n. 5, p. 378-385, 2014.

SANTOS, J. P. M. et al. Análise da funcionalidade de idosos com osteoartrite. Fisioter. Pesq., v. 22, n. 2, p. 161-168, 2015.

SILVA, A. R. et al. Doenças crônicas não transmissíveis e fatores sociodemográficos associados a sintomas de depressão em idosos. J. Bras. Psiquiatr. v. 66, n. 1, p. 45-51, 2017.

SOCIEDADE BRASILEIRA DE CARDIOLOGIA (SBC). 7a. Diretriz Brasileira de Hipertensão Arterial. DBHA. Arq Bras Cardiol., v. 107, n. 3, Supl. 3, p. 1-83, 2016.

SOUZA, I. F. S. et al. Idosos com osteoartrite de joelhos devem realizar avaliação nutricional: revisão integrativa da literatura. Einstein, v. 15, n. 2, p. 226-232, 2017.

VIEIRA, W. H. B. et al. Eficácia do exercício em grupo em pacientes com osteoartrose: estudo piloto quase-experimental. ConScientia e Saúde, v. 11, n. 3, p. 391-400, 2012.

WOLFF, R. B. et al. Aspectos moleculares dos esteroides sexuais sobre a cartilagem e os ossos. Rev. Assoc. Med. Bras., v. 58, n.4, p. 493-497, 2012. 